\title{
Conception, Implementation, and Integration of Heterogenous Information Technology Infrastructures in the Qatar Biobank
}

\author{
Eleni Fthenou, Ameena Al Emadi, Fahad F. Mahal, \\ Leo T. Chettupuzhakaran, Asma Al Thani, ${ }^{1-4}$ and Nahla Afifi ${ }^{1}$
}

The overall goal of the Qatar Biobank (QBB) is to collect, manage, and distribute high-quality human biospecimens with appropriate clinical and/or research annotation and associated phenotypic data, aiming to be an important and essential resource of medical research and evidence-based health care system policies in Qatar. To manage and collect large volumes of data, the QBB has been investing in a number of information management solutions, trying to avoid inflexibility of traditional systems and accommodate changes in data sources and workflows. This article aims to present the information technology solutions of QBB based on a free, opensource software solution, considered a reliable alternative to commercial solutions. After evaluating the free, opensource software solutions available for biobanks, Onyx from ObiBa was utilized to develop custom components to interface various clinical devices, LIMS and Picture Archiving and Communication System, which has varying integration capabilities. This is a showcase for biobanks to carefully evaluate and select hardware and software to automate their operations providing the functions required for business continuity.

Keywords: Qatar Biobank, IT infrastructure, data integration

\section{Introduction}

S INCE 2012, THE Qatar Biobank (QBB) has collected $\checkmark$ biological samples and health data from adult ( $\geq 18$ years of age) men and women, Qatari or long-term residents $(\geq 15$ years living in Qatar), and follows up on these participants every 5 years. ${ }^{1,2}$ At present, QBB has enrolled more than 25,000 participants and is aiming to reach a total of 60,000 participants. QBB acts as Qatar's National research platform, providing the scientific community with biological samples and annotated data (i.e., phenotypic, clinical, multiomic data) encouraging practices of collaboration that hold the potential to invent novel health care interventions, improving our knowledge on the development of human diseases. $^{2}$

In addition to the data and sample repository, QBB supports researchers by providing facilities services (i.e., examination rooms), analysis services (i.e., DNA and RNA extraction and Complete Blood Count (CBC) analysis), storage services (i.e., $-80^{\circ} \mathrm{C}$, liquid nitrogen), and information technology (IT) services (i.e., customized solutions) according to the needs of different research initiatives. ${ }^{2}$
Modern biobanking has become an essential part of biomedical research. ${ }^{3,4}$ The mission of QBB is to manage and distribute high-quality biospecimens with appropriate clinical and/or research annotation and the associated phenotypic data to its stakeholders. Moreover, QBB is linked with Qatar's national health care system to exchange information either by receiving medical data or by referring participants to the Hamad General Hospital. In this context, an efficient, robust, and scalable computing or informatics systems should be in place to support the various key functionalities of QBB.

At the time QBB was established, there were limited available IT solutions for biobanking. Acknowledging the importance of the IT department, the QBB invested in it by hiring many new staff. The QBB IT team then developed a dynamic information infrastructure consisting of heterogeneous solutions using open-source, in-house developed, commercial, and customized solutions to support the processes and workflows between QBB and internal departments and/or external stakeholders.

This article aims to outline the current QBB IT infrastructure and describe our approach to data integration, methods for data extraction, de-identification, and the consolidation of

\footnotetext{
${ }^{1}$ Qatar Biobank, Qatar Foundation for Education, Science, and Community, Doha, Qatar.

${ }^{2}$ College of Health Sciences, Qatar University, Doha, Qatar.

${ }^{3}$ Biomedical Research Center, Qatar University, Doha, Qatar.

${ }^{4}$ Qatar Genome Program Committee, Doha, Qatar.
} 
data in a shared data model, the data marts, and abstraction layers, as well as the query system for the researchers; and discuss the major challenges to build and maintain such infrastructure.

\section{QBB IT Infrastructure Design and Implementation}

\section{Conceptual basis of QBB IT infrastructure}

The hardware and software at QBB support processes such as the management of large data, and the maintenance of the privacy and confidentiality of the participants, facilitates efficiencies in the laboratory, and enables the management of multi-omics data, as well as data integration with the Qatar national health care system. QBB Informatics team designed the IT infrastructure by first delineating critical requirements to meet departmental expectations. The conceptual basis of the QBB IT infrastructure design is presented in Figure 1. QBB operates within two different networks; the Clinic network that is a closed (air gapped), secured network and the Admin network that is open, exposed to the internet, managing communication and data integration with external systems (Fig. 1). This design is built to comply with the QBB data management and information security policy, minimizing the risk of a breach of sensitive information. More specifically, the Clinic network encompasses systems such as the production application servers of clinic and laboratory, the Production (Active Directory) AD Servers and Files sharing Server, production databases, production Picture Archiving and Communication System (PACS) servers, the Clinic network management system, development/test servers for clinic/laboratory systems, Cantab server (cognition function application), Automated Biorepository server, PACS for magnetic resonance imaging (MRI) and ultrasound, Clinic personal computers (PCs) and Clinic Instruments, DICOM modalities, and Laboratory PCs and instruments. The Admin network incorporates the production systems such as temperature monitoring solutions, Door Access, File Server and Active Directory (users access management), Participant Appointment Management System (PAMB), Development PCs used by system developers, MRI modality, Automated Biostore, connection to health care providers (i.e., Hamad General Hospital and Sidra hospital) through Qatar Government Network, and the Test and Development Servers for the various applications. The access to internet and shared services is restricted to administration PCs, Wireless networks, and iPads through MPLS line (Bandwidth $50 \mathrm{Mbps}$ ) to Qatar Foundation Headquarter (Fig. 1).

\section{QBB IT department structure, competencies, and role}

The biobanking landscape is continuously evolving from local biorepositories to robust organizations. ${ }^{3,5-7}$ Likewise, QBB has been evolving over the years by using highthroughput technologies, adding different types of biospecimens in the data repository to meet the requirements of the different research initiatives in sample collection, processing, and storage, handling large amount of data, ensuring compliance with ethical laws, policies, and standards, and providing data sharing services. The QBB IT department consists of several staff with distinct roles (Table 1) to support QBB business continuity and evolution.

\section{QBB hardware and basic software}

Key components for the success of the QBB IT infrastructure are the proper operating systems to run the software with sufficient memory and acceptable processor speed for efficient operations. As the QBB research platform grows, system and integration needs are evolving as well, requiring greater processing power and extra storage capacity. Table 2 describes the current hardware and basic software specifications that support the QBB IT infrastructure. The QBB operating system was selected based on its broad compatibility with the systems used or potentially to be used. An important element of the QBB IT infrastructure for the protection of sensitive participant information is the security software against malware. Security systems and processes for regular scheduled system scans and updates are in place to protect QBB data from malware, including viruses, spyware, adware, and ransomware. In addition, hardware firewalls in the two different networks (Clinic and Admin) are in place to monitor network traffic and when malicious actions are detected, data transmission is blocked (Fig. 1). Another main component for QBB data integrity is the system and data backup. QBB system backs up data through Direct Attached Storage and Network Attached Storage in physical storage forms. No cloud storage is used to minimize the possibility of a data breach and in compliance with Qatari Government Regulation (Data Management Policy). ${ }^{8}$ The primary backup data set up is located at QBB facilities, including servers, storages, and backup tapes.

Periodically encrypted backup tapes are transferred outside the QBB building and stored in a secured environment within a fire safe cabinet. Furthermore, QBB established a secondary data center site to support business continuity in case of primary data center site failure. The data center is built on a virtualization environment to ensure optimal usage of IT hardware resources and fast response to business requirements.

\section{QBB application landscape: functional components and integrations}

The core of the QBB IT infrastructure consists of four main functional components: (1) Data management, (2) biorepository management, (3) reporting system, and (4) participant management. All functional components are critical for the QBB business continuity and success. These functional areas are operated by various distinct software applications with a high degree of integration between them. Figure 2 presents the QBB application landscape and integration level between the distinct internal and external applications. Table 3 describes the different software applications, scope, and sources. The QBB data management supported by the IT infrastructure links thousands of participants with more than 2.5 million biological samples, and clinical data and medical records data (Diagnosis Records) that are integrated through Qatar's national health care system. The QBB Clinical Information System (CIS) is located within the Clinic network and tracks participant registration, informed consent forms, data collection through questionnaires software, and physical measurements through Onyx system (Fig. 2) by CIS through a two-way communication. This is integrated with the Clinical Device Software (CDS) using Onyx integration library to exchange the information captured from the different device 


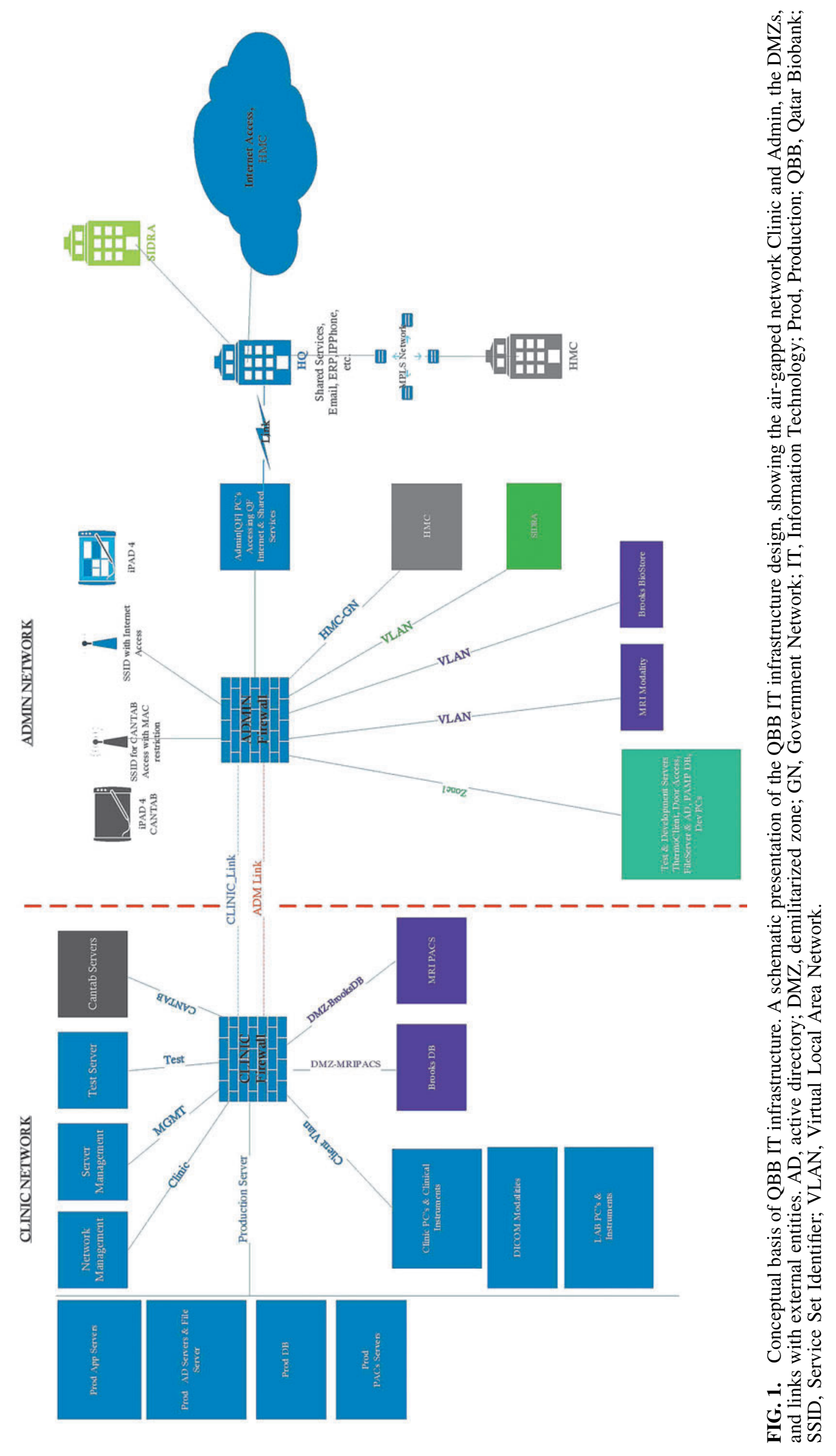


Table 1. Qatar Biobank Information Technology Team Competencies and Role Description

\begin{tabular}{|c|c|c|}
\hline Role & Number & Description \\
\hline Infrastructure administrator & 1 & $\begin{array}{l}\text { Network configuration and performance monitoring } \\
\text { Manage QBB Data Center facility } \\
\text { Ensure network availability and security } \\
\text { Monitor QBB storage availability } \\
\text { Ensure network availability } \\
\text { Configure network security zones and integration management }\end{array}$ \\
\hline System administrator & 1 & $\begin{array}{l}\text { Virtual servers configurations } \\
\text { Upgrade Operating System to latest version } \\
\text { System Security Patching } \\
\text { System backup and restoration validation and testing } \\
\text { Installation and configuration of operating system } \\
\text { System performance monitoring and fine tuning } \\
\text { Manage system migration } \\
\text { Manage end users' access rights and permissions }\end{array}$ \\
\hline $\begin{array}{l}\text { Clinical information system } \\
\text { specialist }\end{array}$ & 1 & $\begin{array}{l}\text { Clinical systems integration design } \\
\text { Manage IT project and ensure end users' requirements are implemented } \\
\text { correctly } \\
\text { Participate in quality assurance and application testing before Go-Live } \\
\text { Develop Business Requirements document }\end{array}$ \\
\hline Data base developer & 1 & $\begin{array}{l}\text { Develop scripts for data extracts to be provided to the researchers } \\
\text { Quality assurance and review of data extract before submission to the } \\
\text { researchers } \\
\text { Control QBB data catalogue }\end{array}$ \\
\hline Data base administrator & 1 & $\begin{array}{l}\text { Plan and implement for data migration } \\
\text { Monitor data base performance } \\
\text { Develop data marts for data extract requests } \\
\text { Database backup and restoration validation and testing } \\
\text { Manage and control access to QBB data bases }\end{array}$ \\
\hline Application developer & 2 & $\begin{array}{l}\text { Develop new applications to support business performance } \\
\text { Enhance and bug fixing } \\
\text { Customize open-source solutions used by clinic and laboratory } \\
\text { Manage application versioning and releases } \\
\text { Application deployment on testing and production environment } \\
\text { User acceptance testing }\end{array}$ \\
\hline Technical support & 2 & $\begin{array}{l}\text { Support QBB end users (Admin, Clinic, and Laboratory) } \\
\text { Resolve technical issues related to PCs connected to the clinic devices } \\
\text { Provide end users training for in-house developed systems } \\
\text { Escalate unresolved issues to second level of support (Application, } \\
\text { Infrastructure, Data Base) } \\
\text { Coordinate with third party suppliers if required }\end{array}$ \\
\hline
\end{tabular}

IT, Information Technology; PCs, personal computers; QBB, Qatar Biobank.

Table 2. Sample of QAtar Biobank Clinic AND Admin Servers Specifications

\begin{tabular}{llll}
\hline Server & CPU & Memory & \multicolumn{1}{c}{ Disk } \\
\hline Clinic Onyx Data Base & 4Core & $32 \mathrm{~GB}$ & 1.1TB SSD \\
$\quad$ Production & & & $750 \mathrm{~GB}$ \\
Clinic Onyx Application & 4Core & $32 \mathrm{~GB}$ & $200 \mathrm{~GB} \mathrm{SSD}$ \\
PACS & 4Core & $8 \mathrm{~GB}$ & $6 \mathrm{~TB}$ \\
CANTAB & 6Core & $180 \mathrm{~GB}$ & $16 \mathrm{~GB}$ \\
Active Directory & 2Core & $8 \mathrm{~GB}$ & $100 \mathrm{~GB}$ \\
Backup & 2Core & $4 \mathrm{~GB}$ & $3 \mathrm{~TB}$ \\
LIMS Data Base & 4Core & $16 \mathrm{~GB}$ & $100 \mathrm{~GB}$ \\
& & & $500 \mathrm{~GB} \mathrm{SSD}$ \\
Admin Test Application & 2Core & $8 \mathrm{~GB}$ & $80 \mathrm{~GB}$ \\
\hline
\end{tabular}

CANTAB, Cambridge Neuropsychological Test Automated Battery; LIMS, Laboratory Information Management System; PACS, Picture Archiving and Communication System. software systems to the participant level (Fig. 2). CDS is integrated (two way) with the PACS providing convenient access to images from the relevant devices (Ultrasound machines, iDXA, and so on). An important component of the data management functional area is the integration of the CIS with the Laboratory Information Management System (LIMS) and the Medical Review System (MRS). LIMS is the main system for sample management and is integrated with the Laboratory Device Software, including all automated systems within the laboratory environment (i.e., Brooks automation biostore, Fluidigm, and flow cytometry) (Fig. 2). MRS is an application developed in-house allowing the QBB medical review office the ability to view and evaluate the results of certain measurements, laboratory results, and participant medical, as well as their family medical history. MRS also supports reporting on participants and referral to Hamad Medical Corporation (Fig. 2). Participant management is based on two systems, the participant management system of the actual QBB participants (consented to participate in the study) is hosted in the Clinic network and the system to manage the potential participants 


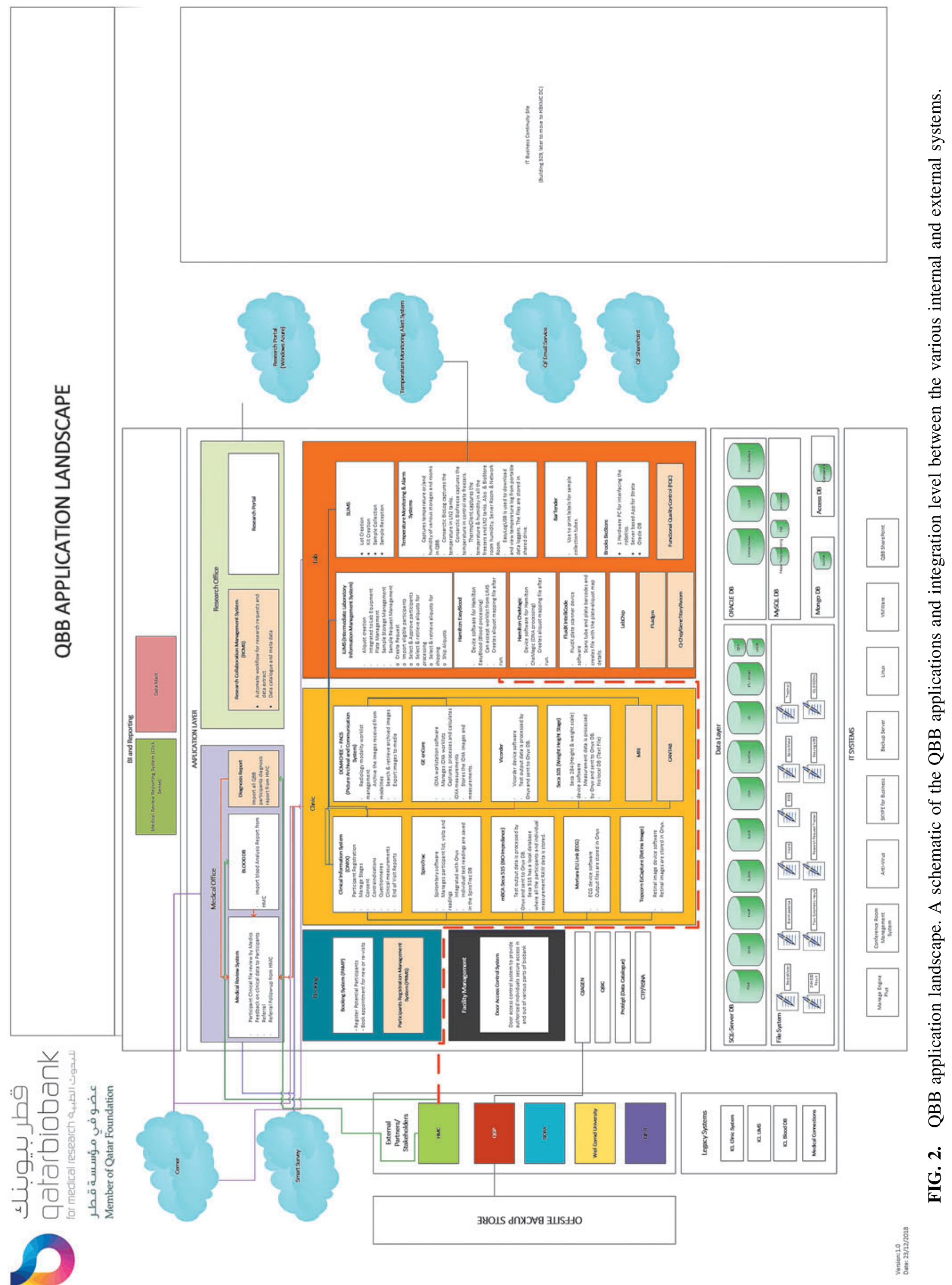


Table 3. QATar Biobank Information Technology Infrastructure Systems

\begin{tabular}{|c|c|c|}
\hline System & Scope & Source \\
\hline PAMP_Booking System & $\begin{array}{l}\text { Register potential participants } \\
\text { Book appointment for new/revisits }\end{array}$ & Commercial \\
\hline $\begin{array}{l}\text { Onyx (Clinical Information } \\
\text { System) }\end{array}$ & $\begin{array}{l}\text { Participant registration } \\
\text { Manage stages } \\
\text { Consent } \\
\text { Contraindications } \\
\text { Questionnaires } \\
\text { Clinical measurements } \\
\text { End-of-visit reports }\end{array}$ & $\begin{array}{l}\text { Open source, in-house } \\
\text { customized } \\
\text { and developed }\end{array}$ \\
\hline Medical Review System & $\begin{array}{l}\text { Participant clinical file review by medics } \\
\text { Feedback on clinical data to participants } \\
\text { Referral } \\
\text { Referral follow-up from HMC }\end{array}$ & In-house built \\
\hline $\begin{array}{l}\text { Blood DB (Laboratory } \\
\text { Analysis Reports) }\end{array}$ & $\begin{array}{l}\text { Blood analysis report from HMC } \\
\text { View and print results }\end{array}$ & In-house built \\
\hline LIMS & $\begin{array}{l}\text { Sample collection } \\
\text { Lot management } \\
\text { Kit management } \\
\text { Tube labeling } \\
\text { Aliquot creation } \\
\text { Integrated to laboratory equipment } \\
\text { Plate management } \\
\text { Sample storage management } \\
\text { Sample request management } \\
\text { Create request } \\
\text { Import eligible participants } \\
\text { Select and approve participants } \\
\text { Select and retrieve aliquots for processing } \\
\text { Select and retrieve aliquots for shipping } \\
\text { Ship aliquots }\end{array}$ & In-house built \\
\hline PACS & $\begin{array}{l}\text { Radiology modality worklist management } \\
\text { Archive the images received from modalities } \\
\text { Search and retrieve archived images } \\
\text { Export images to media }\end{array}$ & Open source \\
\hline GE encore & $\begin{array}{l}\text { iDXA workstation software } \\
\text { Manages iDXA worklists } \\
\text { Captures, processes, and calculates iDXA measurements } \\
\text { Stores the iDXA images and measurements. }\end{array}$ & Commercial \\
\hline SpiroTrac & $\begin{array}{l}\text { Spirometry software } \\
\text { Manages participant list, visits, and readings } \\
\text { Integrated with Onyx } \\
\text { Individual test readings are saved in the SpiroTrac DB }\end{array}$ & Commercial \\
\hline $\begin{array}{l}\text { Temperature Monitoring } \\
\text { and Alarm Systems }\end{array}$ & $\begin{array}{l}\text { Captures temperature or/and humidity of various storages } \\
\text { and rooms in QBB. } \\
\text { Consarctic BioLog captures the temperature in LN2 tanks. } \\
\text { Consarctic BioFreeze captures the temperature in control } \\
\text { rate freezers. } \\
\text { ThermoClient captures the temperature and humidity in all } \\
\text { the freezers and LN2 tanks. Also, BioStore room } \\
\text { humidity, server room, and network room. } \\
\text { EasyLogUSB is used to download and view temperature log } \\
\text { from portable data loggers. The files are stored in shared } \\
\text { drive }\end{array}$ & Commercial \\
\hline SmartSurvey (Online Survey) & $\begin{array}{l}\text { Participant total visit feedback survey after medical review. } \\
\text { Cloud solution } \\
\text { Data can be downloaded in various formats }\end{array}$ & Commercial \\
\hline Door Access Control System & $\begin{array}{l}\text { Door access control system to provide authorized } \\
\text { individuals secure access in and out of various parts of } \\
\text { biobank }\end{array}$ & Commercial \\
\hline Hamilton EasyBlood & $\begin{array}{l}\text { Device software for Hamilton EasyBlood (Blood } \\
\text { processing) } \\
\text { Can accept worklist from LIMS } \\
\text { Creates aliquot mapping file after run. }\end{array}$ & Commercial \\
\hline
\end{tabular}


TABle 3. (CONTINUED)

\begin{tabular}{|c|c|c|}
\hline System & Scope & Source \\
\hline Hamilton CheMagic & $\begin{array}{l}\text { Device software for Hamilton CheMagic (DNA processing) } \\
\text { Creates aliquot mapping file after run }\end{array}$ & Commercial \\
\hline FluidX IntelliCode & $\begin{array}{l}\text { FluidX plate scanner device software } \\
\text { Scans tube and plate barcodes and creates file with the plate- } \\
\text { aliquot map details }\end{array}$ & Commercial \\
\hline BarTender & Use to print labels for sample collection tubes & Commercial \\
\hline Vicorder & $\begin{array}{l}\text { Vicorder device software } \\
\text { Test output data are processed by Onyx and sent to Onyx } \\
\text { DB }\end{array}$ & Commercial \\
\hline mBCA Seca 515 & $\begin{array}{l}\text { Bioimpedance device software } \\
\text { Test output data are processed by Onyx and sent to Onyx } \\
\text { DB. } \\
\text { Seca } 515 \text { has a local database where all the participants and } \\
\text { individual measurement RAW data are stored }\end{array}$ & Commercial \\
\hline Seca 101 & $\begin{array}{l}\text { Seca } 284 \text { (height and weight scale) device software } \\
\text { Measurement data are processed by Onyx and sent to Onyx } \\
\text { DB. } \\
\text { No local DB }\end{array}$ & Commercial \\
\hline Mortara ELI Link & $\begin{array}{l}\text { ECG device software } \\
\text { Output files are stored in Onyx }\end{array}$ & Commercial \\
\hline Topcorn EzCapture & $\begin{array}{l}\text { Retinal Image device software } \\
\text { Retinal images are stored in Onyx. }\end{array}$ & Commercial \\
\hline PRMS (Participant Portal/CRM) & $\begin{array}{l}\text { Online portal for participants and potential participants } \\
\text { Book appointments online } \\
\text { Download results online }\end{array}$ & $\begin{array}{l}\text { Commercial } \\
\text { customized }\end{array}$ \\
\hline RCMS & $\begin{array}{l}\text { Research collaboration portal } \\
\text { Data catalogue } \\
\text { Data extract and query system }\end{array}$ & $\begin{array}{l}\text { Commercial } \\
\text { customized }\end{array}$ \\
\hline
\end{tabular}

DB, database; ECG, electrocardiogram; HMC, Hamad Medical Corporation; RCMS, Research Collaboration Management System.

and their appointments is hosted in the Admin network. More specifically, Admin network facilitates all the applications connected outside the QBB facilities. The participant appointment scheduling application (PAMP Booking) manages participant booking and appointment (through QBB website or phone call), while the participant registration is managed through the CIS system (Fig. 2). The Admin network supports temperature monitoring systems (hosted on the cloud), integration with Qatar national health care system (Cerner), Online Feedback Surveys, Research Portal, and Qatar Foundation services, that is, e-mail, Enterprise Resource Planning (ERP), and SharePoint portal (Fig. 2).

The Data management component starts with data collection on the clinic side, and these data are stored in secure access-controlled database management systems, where critical changes (such as edit or delete) of operational data within the CIS are being tracked using audit trail functionality with timestamp. When researchers make a request for certain groups of data, they are retrieved from the data mart and anonymized before handing it over for research use. This anonymization also applies to different types of images type (retinal images, ultrasound, etc.). The method/tool used for anonymization is based on the type of data that are to be anonymized. For example, for radiology images, QBB uses CTP/RSNA toolset to de-identify PII.

The Medical office in QBB can retrieve participants' reports through MRS; they update the status of participants' review process back to the system (i.e., referrals to Hamad Medical Corporation (HMC)). Medical office performance is being measured through business intelligence dashboard, which shows various statistical charts and reports on the clinical re- view processes. The business intelligence tool depends on centralized data base that aggregates data from the live system of MRS and CIS on daily basis (at Night) in order not to disturb performance of the operational system. The Biostore facility, Liquid Nitrogen tank, freezer, and room temperatures within the QBB are monitored through a cloud-based temperature monitoring service, where the laboratory staff can monitor the live temperature data on their mobile phones. An automatic phone call alert is sent to the responsible team in the case of a temperature drop. Samples are collected and managed through a laboratory information system developed in-house and a set of laboratory devices that are integrated with it. The integration is accomplished through files or databases (DBs) depending on the integration method supported by different devices. The same applies to CIS and clinical instruments and $95 \%$ of the data generated by clinical devices are stored into data base management systems in a structured format, while the remaining $5 \%$ is stored as received from the clinical devices.

The QBB applies both physical and digital security control. Access to the QBB data center is restricted to authorized personnel and controlled through access card managed by a Door Access Control System. Access to clinic, laboratory, and medical office systems is restricted to authorized users where a different level of privilege is granted to the users depending on their role (Normal users, Supervisor, and IT Admin). Login to the network to access the applications is managed through Operating System user's credentials and is managed and controlled through the application of Active Directory password protection policies.

Data extraction for the researchers is manually processed through an in-house developed data mart system, which 
Table 4. Qatar Biobank Information System Review Against ISBER Information System Evaluation Checklist

\begin{tabular}{|c|c|c|}
\hline Information system evaluation checklist & Yes/No & Explanatory notes \\
\hline \multicolumn{3}{|l|}{ Controlled vocabulary and data integrity features } \\
\hline $\begin{array}{l}\text { System provides ability to maintain controlled } \\
\text { vocabularies (ontologies) to enforce data } \\
\text { standardization and control. }\end{array}$ & Yes & $\begin{array}{l}\text { QBB uses international or national standard vocabularies } \\
\text { wherever applicable, such as SPREC to characterize } \\
\text { biospecimen, LOINC for Laboratory Tests, and ISO- } \\
3166 \text { for nationalities. QBB data catalogue is available } \\
\text { for group of data sets such as questionnaires, images, } \\
\text { and laboratory results. QBB is in process of building a } \\
\text { comprehensive data catalogue using a structured } \\
\text { prebuilt solution through open-source ontology editor } \\
\text { to control data catalogue versions. }\end{array}$ \\
\hline $\begin{array}{l}\text { System provides users with intuitive on-demand } \\
\text { access to data and the ability to represent the data } \\
\text { (for view, file export, or print) in a variety of } \\
\text { formats without knowledge of proprietary code. }\end{array}$ & Yes & $\begin{array}{l}\text { Self-registration features are available in the systems } \\
\text { that are public facing such as participants and } \\
\text { researchers' portals. For reporting and data analysis } \\
\text { purposes, QBB has implemented a user-friendly self- } \\
\text { service dashboard solution where the end users can } \\
\text { build their reports without any technical knowledge. } \\
\text { For instance, this solution is used by Medical Review } \\
\text { Team to access and analyze the data relevant to the } \\
\text { Medical Review processes. }\end{array}$ \\
\hline \multicolumn{3}{|l|}{ Security, privacy, and auditing features } \\
\hline $\begin{array}{l}\text { System is capable of maintaining user profiles and } \\
\text { credentialing users for different levels of access and } \\
\text { functionality. }\end{array}$ & Yes & $\begin{array}{l}\text { Application systems are capable of enforcing fine- } \\
\text { grained access control policy across all users. For each } \\
\text { system, there are built-in user management modules } \\
\text { that control user access to various functionalities } \\
\text { based on the roles granted to them. Enterprise level } \\
\text { user profiling is managed through Active Directory } \\
\text { solution. }\end{array}$ \\
\hline $\begin{array}{l}\text { System provides audit trail that minimally tracks user } \\
\text { ID, date, and content of change at the field level }\end{array}$ & Yes & $\begin{array}{l}\text { Audit of changes is enabled in critical information } \\
\text { systems such as Clinical information system, } \\
\text { Laboratory system, and medical review systems. In } \\
\text { the audit trail, along with the field level data, } \\
\text { usernames, date and time, and IP address are also } \\
\text { being tracked for reference. }\end{array}$ \\
\hline \multicolumn{3}{|l|}{ Subject management features } \\
\hline $\begin{array}{l}\text { Data elements pertaining to each subject are } \\
\text { sufficiently extensive or extensible (e.g., the ability } \\
\text { to maintain demographic attributes, including race, } \\
\text { ethnicity, date of birth, and gender)? }\end{array}$ & Yes & $\begin{array}{l}\text { Participant demographics are maintained in the clinical } \\
\text { information system, and linked to other systems such } \\
\text { as LIMS and PACS. In QBB subject registration } \\
\text { module, the subject attributes can be configured based } \\
\text { on study requirements and can be extended to include } \\
\text { new attributes. For example, for QBIC Study, we } \\
\text { capture additional information (about spouse) during } \\
\text { registration, which is not captured for other studies. }\end{array}$ \\
\hline $\begin{array}{l}\text { System provides ability to track and manage events } \\
\text { associated with a particular subject and date (e.g., } \\
\text { visit, donation, and examination) }\end{array}$ & Yes & $\begin{array}{l}\text { Multiple events related to participants and or } \\
\text { participants' biological samples are tracked in the } \\
\text { clinical information system, participants' portal, and } \\
\text { laboratory system. In case of feedback visits and } \\
\text { referrals, the information is maintained in the Medical } \\
\text { Review System and Booking system. System keeps } \\
\text { record of various events associated to individual } \\
\text { subjects, as well as uses the data to ensure that the } \\
\text { subject events conform to study protocol. For } \\
\text { instance, in QBB Cohort study, follow-up visit is } \\
\text { allowed only after } 5 \text { years of completing the baseline } \\
\text { visit. During subject visit registration event, system } \\
\text { ensures that subject has completed } 5 \text { years. }\end{array}$ \\
\hline
\end{tabular}


TABle 4. (CONTINUED)

\begin{tabular}{|c|c|c|}
\hline Information system evaluation checklist & Yes/No & Explanatory notes \\
\hline Provides ability to associate a subject with a study & Yes & $\begin{array}{l}\text { Participant contribution to different studies is being } \\
\text { linked through the clinical information system, } \\
\text { Laboratory system, and medical review system. The } \\
\text { underlying design supports one subject to be linked to } \\
\text { one or more studies. During enrolment to a study, } \\
\text { system also ensures that the subject meets the study } \\
\text { inclusion/exclusion criteria. }\end{array}$ \\
\hline $\begin{array}{l}\text { Provides ability to assign system-generated unique } \\
\text { subject identifier }\end{array}$ & Yes & $\begin{array}{l}\text { Each participant has a unique ID generated by the } \\
\text { system along with the National ID. }\end{array}$ \\
\hline Provides ability to manage subject de-identification. & Yes & $\begin{array}{l}\text { System has the capability to manage de-identification of } \\
\text { subjects to ensure that the data shared neither identify } \\
\text { nor provide a reasonable basis to identify an } \\
\text { individual. The method/tool used for de-identification } \\
\text { is based on the type of data that are to be de-identified. } \\
\text { For example, for radiology images, QBB uses } \\
\text { CTP/RSNA toolset to de-identify PII. }\end{array}$ \\
\hline
\end{tabular}
System provides ability to generate/assign a unique accession to each sample.

System provides ability to define an unlimited number of samples: whole-blood, tissue, cellular lysates, DNA, RNA, proteins, etc.

System provides ability to assign sample component with physical location.

System provides ability to associate the sample and component to the participant.

System provides ability to annotate sample with sample attributes, like method of specimen preparation and environmental conditions under which specimen is stored (e.g., type of sample, volume, container size, description, date drawn, source of sample, person storing, and temperature).

System provides ability to a user with special permissions to define hierarchical storage configurations.

System provides ability to track specimens with barcoded IDs printed on labels.

Provides ability to query/search inventory of specimen and specimen components.

System maintains sample genealogy on aliquots, derivative, and pooled samples (e.g., DNA derived from PBMC derived from whole blood)
Yes Each sample has a unique sample ID. LIMS generates the unique Sample ID for samples such as collection samples and pooled sample. For samples in prebarcoded containers (e.g., aliquot containers), LIMS allows to assign the barcode and ensures that the barcode is unique.

Yes The laboratory information system supports unlimited number of sample types per participant. However, to ensure that the number and type of samples created conform to the study protocol, system has the capability to define rules and restrictions in the system.

Yes The laboratory information system supports linkage between each sample and its associated physical location. Movement of sample between physical location is also tracked in the system.

Yes The laboratory information system supports the association between the sample, components, and the participant.

Yes The laboratory information system implemented in QBB stores various attributes per sample such as type of sample, volume, container size, description, date drawn, source of sample, person storing, temperature, and instruments details. System is extendable to introduce new annotation attributes.

Yes Laboratory information system and Brooks Automated Storage System provide the ability to define hierarchical storage configurations for authorized users. System currently manages storages of different hierarchical layouts, which include automated storage system, LN2 Tanks, Freezers, Refrigerators, etc.

Yes System can track samples with barcode printed on label as well as barcode printed on the container itself.

Yes Laboratory information system and Brooks Automated Storage System provide ability to generate reports related to the inventory of biological samples and sample types. Authorized users can search inventory of samples based on various search criteria, including sample attributes, storage attributes, and subject attributes.

Yes Laboratory information system maintains sample genealogy on aliquots, derivative, and pooled samples. Each aliquot/sample can be tracked back to the source sample(s). 
TABle 4. (CONTINUEd)

\begin{tabular}{|c|c|c|}
\hline Information system evaluation checklist & Yes/No & Explanatory notes \\
\hline $\begin{array}{l}\text { System provides logistics management and chain-of- } \\
\text { custody tracking (e.g., shipping and receiving) }\end{array}$ & Yes & $\begin{array}{l}\text { LIMS automatically tracks the chain of custody for each } \\
\text { action performed on a sample throughout that } \\
\text { sample's life cycle. LIMS maintains information on } \\
\text { various processes in Laboratory such as sample } \\
\text { collection, receiving, processing, storing, and shipping } \\
\text { to allow sample tracking in all different stages of } \\
\text { biobanking. }\end{array}$ \\
\hline $\begin{array}{l}\text { System provides ability to configure rules or } \\
\text { restrictions to manage access to specimen } \\
\text { information. }\end{array}$ & Yes & $\begin{array}{l}\text { Laboratory information system and Brooks Automated } \\
\text { Storage system provide the ability to configure rules } \\
\text { and restrictions depending on the role and access level } \\
\text { permissions of a user to access specimen information. } \\
\text { For example, samples cannot be released without } \\
\text { laboratory manager approval. Rules and restrictions } \\
\text { can be configured in the system as per the } \\
\text { study/organizational protocol. System validates and } \\
\text { ensures that only the users with right permission can } \\
\text { access the sample information. }\end{array}$ \\
\hline $\begin{array}{l}\text { System provides mechanism for maintaining } \\
\text { specimen lifecycle and disposition (e.g., system } \\
\text { tracks amount used and decrements from available } \\
\text { amount). }\end{array}$ & Yes & $\begin{array}{l}\text { System uses defined rules and configurations to ensure } \\
\text { that specimen lifecycle is as per the study protocol. } \\
\text { System manages the samples status and other relevant } \\
\text { information with each sample process. For example, } \\
\text { based on configurations, system automatically adjusts } \\
\text { the volume of parent sample when a child aliquot is } \\
\text { prepared. }\end{array}$ \\
\hline $\begin{array}{l}\text { System provides ability to create and maintain } \\
\text { complex queries/searches using associated subject }\end{array}$ & Yes & $\begin{array}{l}\text { The system provides advanced search capabilities where } \\
\text { inclusion/exclusion criteria can be applied. }\end{array}$ \\
\hline
\end{tabular}
attributes

Analytics and reporting features

Provides the user with a defined process for creating queries/searches on data in the system.

Provides ability to create and maintain reports as standard reports that can be selected by a user from a list

Provide the user with the ability to define ad hoc queries/searches and custom reports in common terms, without requiring knowledge of proprietary code.

Provide the user with the ability to save queries/searches for future reuse.

System provides ability to export data in delimited formats: .csv, or .xls and XML

Technical features

System has an integrated database. Describe the DBMS that is most commonly used with the System.

Yes

QBB has a defined data extraction process in place, where data are extracted from a data mart system; in addition, QBB is in process of improving data extraction process to be fully automated through research collaboration management system.

Yes Standard reports are provided in individual systems and users with access permission can run the report after applying filter criteria. Each group of users has different level of access to the reports provided through the clinical information system, Laboratory information system, and Medical Review System.

Yes For reporting purposes, QBB implemented a userfriendly self-service dashboard solution where the end users can build their reports without any technical knowledge. This solution is used by Medical Review Team and Management to access and analyze the data relevant to the Medical Review processes.

Yes The implemented dashboard management system allows the end users to save ad-hoc queries/searches for future use.

Yes Based on the business requirement, option is provided in the systems to export the data in required format. QBB data extracts provided to the researchers or internal users can export them into delimited formats such as CSV and XML.

Yes Most of the critical application systems in QBB use Oracle or MS SQL Server databases. This allow, us to have most of the data sources to be in structured format to facilitate data extraction for research purposes and ensure data integrity such as integration between clinical information system and clinical equipment (iDXA, MRI, Ultrasound, etc.) 
TABle 4. (CONTINUED)

\begin{tabular}{|c|c|c|}
\hline Information system evaluation checklist & Yes/No & Explanatory notes \\
\hline \multicolumn{3}{|l|}{ API interoperability features } \\
\hline $\begin{array}{l}\text { System is capable of interfacing with third-party data } \\
\text { sources, applications, and services. }\end{array}$ & Yes & $\begin{array}{l}\text { For integration with systems, devices, and data sources, } \\
\text { QBB uses various integration methods based on the } \\
\text { integration requirements and integration capabilities } \\
\text { of the systems. To ensure data integrity between } \\
\text { systems, QBB has developed APIs in-house to } \\
\text { facilitate data exchange between in-house developed } \\
\text { systems and commercial out of the box solutions. } \\
\text { QBB also uses Database level integration for sharing } \\
\text { data between systems. For example, subject } \\
\text { information is only stored in one system and all other } \\
\text { associated systems fetch the data whenever required } \\
\text { instead of keeping a copy in individual systems. }\end{array}$ \\
\hline
\end{tabular}

API, application program interface; DBMS, database management system; MRI, magnetic resonance imaging.

reads data from different data sources, including legacy systems, which hold participants' clinical and laboratory tests data. QBB is in the process of automating the process of receiving researchers' requests and allowing them to browse the QBB data catalogue online through a portal. The project is in the planning/implementation stage and would enhance time and quality of service provided by QBB to researchers.

Participants' relationship management is an important focus of the QBB; therefore, to enhance the experience of participants, QBB initiated a new project to develop an online portal where participants can manage their appointments online, access their reports, and withdraw from the studies upon their desire. The portal will be integrated with the clinical system to improve data integrity and accuracy.

In addition to the aforementioned components, QBB utilizes several IT system utilities to manage the data center, end users' security, and communication facilities, such as conference room management system, VMware, a backup system, and Anti-virus software.

\section{Discussion}

Available informatics and computing solutions related to biobank science have emerged and matured over recent years. ${ }^{5,7,9,10}$ Challenges faced while building the QBB IT infrastructure were similar to those faced by similar industries. The main examples were managing QBB scope and finding cost-effective solutions to prevent budget overrun, the decision made to purchase commercially available applications versus in-house built applications, and ensuring system security, usability, performance, and compatibility with other systems.

The principal design feature of the QBB IT infrastructure is to ensure the security and integrity of participant data. In this context, controls for data security are provided in several levels. Two secure network connections (Clinic and Admin, Figs. 1 and 2) are in place to support data transfer between client machine and application servers. Internal staff access to critical systems is controlled through user credentials. This transport level security is facilitated by Digital Certificates to ensure that the data are encrypted on transport when they are accessing QBB services online (through the web). The main challenge QBB faces with this infrastructure design is data integration and real-time data integration between the systems sited in the two different networks. QBB currently manually handles data integration between these two networks. More security measures are applied to facilitate system to system data integration such as redesigning both networks and establishing demilitarized zones that are isolated and positioned between the Internet and the air-gapped network, and upgrading Firewalls sited within QBB IT Next Generation Firewalls. These measures allow QBB extra time to detect and address breaches before they would further penetrate into local networks. QBB is following ISBER Best practices; to evaluate the QBB Information System, we have revised it against the ISBER Information System Evaluation checklist presented in Table 4.

Decisions taken to build in-house or purchase commercial products were reached by performing factor analysis of the cost, data security, maintainability, and supportability. One of the core systems in QBB is the CIS, which collects the phenotypic data of participants on their visit to the QBB clinic. When $\mathrm{QBB}$ decided to replace the legacy CIS for performance and expendability reasons, QBB evaluated many commercial solutions for biobanks. QBB was not able to find any solution that met the functional requirements. Most of the solutions were extension LIMS and mainly lacked flexible and extensible clinical device integration capabilities. QBB also considered an Electronic Medical Records solution and found that it would overkill as it would require bigger team and cost for maintenance and support. QBB then looked into the open-source space and evaluated some of the open-source solutions. After evaluation and a Proof of Concept, QBB decided to implement Onyx application from Obiba. Onyx is a solution built for similar setup and has a good questionnaire module and an extensible framework for device integration. With the support from the Obiba team, legacy CIS was replaced with Onyx. Although Onyx was built for similar institutes, it lacked functionalities that are needed for the Biobank; and QBB's in-house team extended Onyx to introduce the missing functionalities. Our main challenge has been the shortage of IT staff to develop in-house solutions. However, the main advantages of the inhouse built applications are the dynamic maintenance and 
system enhancement based on QBB's ongoing operation. We tried to overcome this by hiring temporary staff or by trying to customize commercial solutions according to our needs.

The main challenge of the readymade commercial biobanking products were either the mismatch between the compliance requirements of $\mathrm{QBB}$ security policies or the compliance with our operational model. QBB has various clinical and laboratory devices that are capable of electronically transferring clinical data. As most of the devices have custom interfacing capabilities and do not support international standards such as American Society for Testing and Materials (ASTM), Health Level Seven International (HL7), interfacing of new devices with the legacy CIS system (commercial), was a challenge. After the introduction of Onyx, as it has a simple and flexible interfacing library, QBB is now capable of interfacing new devices with lesser effort. QBB has developed custom components to interface various clinical devices, LIMS and PACS, which has varying integration capabilities. Migration of data from legacy systems was a challenge due to data quality issues and lack of documentation of the source system database. For new systems, QBB ensures that the data sources are well documented and rigorously tested for data quality issues before going live.

To conclude, we believe that a competent IT department with a flexible and scalable IT system is vital for the biobanking industry. Moreover, ISBER Best Practices are a valuable tool that it can be used as a starting point from biobanks to develop or upgrade their IT infrastructure.

\section{Author Disclosure Statement}

No conflicting financial interests exist.

\section{Funding Information}

No funding was received.

\section{References}

1. Al Kuwari H, Al Thani A, Al Marri A, et al. The Qatar Biobank: Background and methods. BMC Public Health 2015; $15: 1208$.
2. Thani AA, Fthenou E, Paparrodopoulos S, et al. Qatar Biobank cohort study: Study design and first results. Am J Epidemiol 2019;188:1420-1433.

3. Paskal W, Paskal AM, Debski T, et al. Aspects of modern biobank activity-Comprehensive review. Pathol Oncol Res 2018;24:771-785.

4. Quinlan PR, Gardner S, Groves M, et al. A data-centric strategy for modern biobanking. Adv Exp Med Biol 2015; 864:165-169.

5. Boutin N, Holzbach A, Mahanta L, et al. The information technology infrastructure for the translational genomics core and the partners biobank at partners personalized medicine. J Pers Med 2016;6:E6.

6. Kinkorova J, Topolcan O. Biobanks in Horizon 2020: Sustainability and attractive perspectives. EPMA J 2018;9: 345-353.

7. Merino-Martinez R, Norlin L, van Enckevort D, et al. Toward global biobank integration by implementation of the Minimum Information About BIobank Data Sharing (MIABIS 2.0 Core). Biopreserv Biobank 2016;14:298306.

8. Ministry of Information \& Communications Technology (ictQATAR). Data Management Policy. May, 2015. http://www .motc.gov.qa/sites/default/files/data_management_policy.pdf (accessed November 18, 2019).

9. Coppola L, Cianflone A, Grimaldi AM, et al. Biobanking in health care: Evolution and future directions. J Transl Med 2019;17:172.

10. Prokosch HU, Beck A, Ganslandt T, et al. IT infrastructure components for biobanking. Appl Clin Inform 2010;1:419 429.

Address correspondence to: Eleni Fthenou, PhD Qatar Biobank Qatar Foundation for Education, Science, and Community Building 317, Hamad Medical City Doha 9744 Qatar

E-mail: efthenou@qf.org.qa 